\title{
Supply Chains and Global Health: An Imperative for Bringing Operations Management Scholarship into Action
}

\author{
Santiago Kraiselburd \\ Escuela de Negocios, Universidad Torcuato Di Tella, 1010 Calle Sáenz Valiente, Buenos Aires, Argentina, and INCAE Business School, \\ PO Box 960-405, Alajuela, Costa Rica, santiago.kraiselburd@incae.edu \\ Prashant Yadav \\ William Davidson Institute, Ross School of Business, and School of Public Health, University of Michigan, 724 East University Avenue, \\ Ann Arbor, Michigan 48109, USA, yadavp@umich.edu
}

\begin{abstract}
$\mathrm{M}$ any people in developing countries do not have access to effective vaccines, medicines, and other life-saving health technologies. Shortage of health care workers, severe financial constraints, and lack of awareness are some of the major obstacles that prevent higher access. However, ineffective and poorly designed supply chains for purchasing and distributing the medicines, vaccines, and health technologies are one of the most important barriers to increasing access. We argue that the ineffectiveness of the global health supply chain can be attributed largely to: coordination problems across multiple stakeholders with widely divergent objectives, lack of careful supply chain design, and use of myopic operational objectives and metrics. The operations management research community can contribute to improving this by applying existing knowledge to the field of global health delivery and by researching new frameworks of analysis which would then become the cornerstones for policy advice to those who design, operate, or finance these supply chains.
\end{abstract}

Key words: global health supply chain; public health; vaccines; essential medicines History: Received: January 2009; Accepted: September 2010, after 1 revision.

\section{Introduction}

Today, our collective scientific knowledge, technological expertise, and economic resources add up to an impressive arsenal that could be used to reduce the unnecessary human suffering resulting from disease and ill-health. Yet, world-wide, millions of people do not have access to these effective vaccines, medicines, and other life-saving health technologies. It is a sad fact that, every year, around 10 million children under the age of 5 die due to lack of access to simple and affordable interventions (WHO 2008). For example, despite significant progress in the prevention and treatment of HIV/AIDS, it still remains the leading cause of adult death in Africa, reducing life expectancy in highly affected countries. It is estimated (Foster et al. 2006) that almost one-third of the world's population does not have access to essential medicines. In addition to this, each year, personal expenditures on health push more than 100 million people below the poverty line ( $\mathrm{Xu}$ et al. 2007).

Clearly, a key barrier that prevents higher access to life-saving medicines and health technologies is poverty itself. As mentioned, people in low- and middleincome countries (and their governments) often lack the money to purchase vaccines and essential medicines. However, empirical evidence from such varied places as Costa Rica, Sri Lanka, Kerala State in India, and Mongolia has shown that income improvement is not a prerequisite for better health outcomes (Caldwell 1986, 1990, Halstead et al. 1985, Kim and Moody 1992). In fact, health can be improved before income increases, as improved health appears to contribute to economic growth (implying a reverse sign of causality is also in play). Driven by this argument, more than $\$ 27$ billion is spent annually on global health by multi-country financing organizations such as the Global Fund to fight AIDS, TB, and Malaria; the World Bank, UNITAID, Global Alliance on Vaccines and Immunization (GAVI); and bilateral donors such as the US government, the British Government, the Bill \& Melinda Gates Foundation; and others (Ravishankar et al. 2009, Yadav 2010).

Ensuring better global health is obviously a complex challenge that involves disentangling many inter-connected causes and effects. Despite the existence of products, technology, and funds dedicated to certain diseases, medicines for these diseases are still not getting to the target population in the right quantities, at the right prices, at the right time, and in the 
right places. Given this, it is clear that a key barrier to improved global health is the limited capacity in resource-poor countries to procure, manufacture, or distribute medicines, vaccines, and others products and technologies that can improve health outcomes. The average performance of health care supply chains is much poorer in low- and middle-income countries than in OECD nations, and varies substantially between countries, regions, programs, delivery channels, and sub-populations. Performance also may vary considerably over time. For example, it is estimated that the average service level of drugs at public health facilities is less than $25 \%$, and even at private outlets, where products are often unaffordable to most of the population, availability is under 65\% (Cameron et al. 2009). This compares to an average service level of $92.5 \%$ at the point of access in the EU15 (Macarthur 2007). The above clearly demonstrates that the supply chain for global health is extremely sick and deserves careful analysis using existing or newly developed break only as analytical tools. A deeper and structured understanding of the supply chain for medicines in low- and middle-income countries would be key to the development of remedial policy interventions that can improve access to medicines and, consequently, health. As researchers, the supply chains for medicines in many low- and middle-income countries present us with a unique opportunity to chart a course that is based on past learning from the design of health care and other supply chains in OECD countries.

\section{Deficiencies in Global Health Supply Chains and Areas for Research}

To help describe the situation, raise debate, and motivate research in this topic, we suggest three main areas where we believe the contribution of researchers from the field of operations and supply chain management can have the greatest impact. These suggestions are based on (i) a few preliminary studies that were carried out by a team involving the authors in a heterogenous set of three to four low- and middle-income countries, (ii) interviews with multiple stakeholders over the last 2 years, and (iii) extensive review of existing published and practioner literature in this field.

\subsection{Inefficiencies in Global Health Supply Chains Often Result from Coordination Problems across Multiple Stakeholders with Widely Divergent Objectives}

There exists a wide array of structural (and institutional) possibilities in how the supply and distribu- tion chains for medicines are organized in low- and middle-income countries (Attridge and Preker 2000, Govindaraj et al. 2000). Additionally, the agencies involved in financing, policy making, and quality regulation also play an important role in the provision of drugs and medical supplies. Figure 1 illustrates the range of actors involved in financing and physical distribution of drugs and health commodities in lowand middle-income countries. The problem is compounded by the multiplicity of agents at each level: for example, the public sector supply chain of Zambia (not a particularly complex case) shows that 12 main categories of health products were procured using 19 different sources of funding, using 17 different procurement organizations, and over six first-tier warehousing providers (Fundafunda and Yadav 2008). Thus, there is often a complex combination of institutions specializing in funding, manufacturing, import, wholesaling, retailing, and various other auxiliary functions that have to join forces in making the drug available to the end-patient (Attridge and Preker 2000).

Many of the problems observed in the global health supply chains are due to the complex interactions between these multiple stakeholders with often conflicting objectives and scope. Although conflicting objectives are not unique to global health supply chains alone, in this case they often involve intertwined and convoluted social, economic, and political interests. While it can be argued that the main objective of most agents in private supply chains in other industries is profit or utility maximization, the motivation behind some key agents in global health supply chains can be very different. For example, what most donors seek can be summarized as "maximize traceable impact on health for each dollar spent, while minimizing corruption, waste, and risks in general that can lead to bad publicity." Traceability is important for two inter-related reasons: it enables the learning process by facilitating the isolation of causal relationships, and it allows the donors to claim success (which allows the donors to raise more funds and help more people). The problem is that, to keep traceability and to maximize transparency, efficiency, coordination, and economies of scale are usually sacrificed. For example, while pooling of donor funds, pooled purchasing of medicines, pooled transportation services, and pooled inventories can potentially achieve significant savings, it would also conflict with the traceability objectives: once it is all pooled, it is difficult to trace the impact of a particular donor's contribution or to allocate resources based on differing priorities. Similar tensions arise between transparency, equity of access, efficiency, and responsiveness, etc. The role of a supply-chain integrator that coordinates some core functions across firm boundaries 
Figure 1 Physical and Financial Flows in the Supply Chain for Drugs and Health Commodities in Low- and Middle-Income Countries (Only broad level financial flows are depicted. There are other financial flows such as those flowing upstream from the end-patient and social insurance schemes to the manufacturer.)

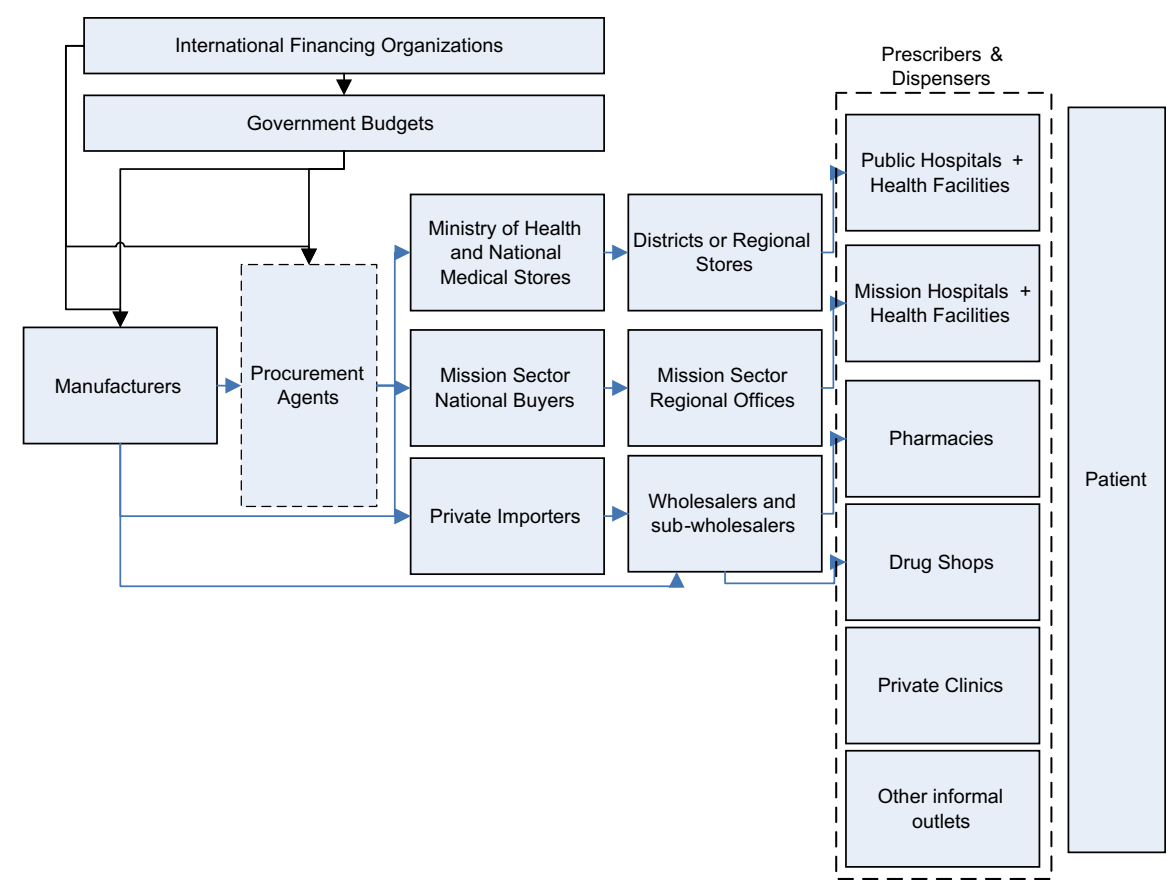

within a supply chain (Parker and Anderson 2002) is currently non-existent in global health supply chains. We conjecture that the lack of a common framework for understanding these supply chains at least in part contributes to this lack of goal congruence and the resulting system-wide inefficiencies. Lack of a common framework of understanding inhibits the development of better incentive alignment mechanisms or new roles in the supply chain. Such a framework needs to be built around a set of hierarchical metrics for all the actors involved. The development of such a framework requires new research as conventional frameworks involving profit maximization or even social welfare maximization do not necessarily apply in this case.

\subsection{Supply Chain Design for Global Health} Requires Careful Context-Specific Analysis Which Is Very Often Lacking

A system for supplying medicines and health commodities can be built using anything from a fully publicly owned to a fully private system. Most OECD countries use a fully privatized system for drug supplies with the government role being limited to quality regulation and contracting. However, in most low- and middle-income countries publicly owned (and operated) central medical stores and autonomous supply agencies are the most commonly used drug supply systems (Seiter 2010). Some countries, such as Tanzania, Uganda, and Zambia, have built their drug supply networks using para-statal entities, whereas others, such as Malawi, have contracted out the procurement, storage, and distribution of essential drugs to international agencies. A plethora of situations exist, and it is not clear what institutional structure works best under what conditions.

Another question that remains unanswered is whether the functions of medicine procurement or distribution should be centralized at the national level or decentralized at the district or the province level. To further compound the complexity of options, public vs. private and centralized vs. decentralized decisions can be combined in different ways. For example, some countries, such as Ghana, have decentralized medicine procurement and distribution and are allowing districts to purchase drugs and supplies from private sector suppliers. It can be argued that allowing district purchases from the private sector creates competition for the public sector supply system and may lead to improving its efficiency. However, it is not clear if the positive impact of competition offsets the loss of scale economies that exist in the public sector supply system. In addition to this, the conventional trade-off between bargaining power efficiencies of centralization and agility and responsiveness of decentralization poses a unique nuance here due to possible differences in transparency within procurement at the central or decentralized level. 
At a more tactical level, it is not clear what is the "right" number of tiers to have in a country's national drug supply system, the "right" number of competitors within each tier, and whether vertical integration across tiers helps or hinders performance. Here, tensions between economies of scale and monopoly power are clearly part of the issue. Regardless of the degree of vertical integration or number of players within layers, in most countries, irrespective of the geographical size, population density or other characteristics of the country, a three-tier system is utilized, with stocking points at the central warehouse, district or regional warehouses and the primary clinic level (Yadav et al. 2011). A recent quasi-randomized study in Zambia found large improvements in product availability by reducing the number of tiers in the supply system (Vledder et al. 2011).

It is also not clear if a single system is best suited for all essential drugs and medical supplies. It may well be that different systems are suited for different categories of drugs and a careful segmentation of the product categories is required before carrying out such analysis.

Although numerous pilot projects are underway to empirically test some of these issues, the world would certainly benefit from more operations and supply chain management researchers joining this debate.

\subsection{Design Decisions for Global Health Supply Chains Are Often Based on Myopic Operational Objectives and Metrics}

Adequate supply chain design should analyze the physical product flow, information flow, and financial flow. However, in a lot of the recent global health initiatives that originated with new (or recently expanded) donors, financial flows play the critical role in design decisions for global health supply chains. Part of the problem is that, as mentioned earlier, supply chains designed around multiple but non-coordinated financing streams often compromise efficiencies in physical or informational flows. In particular, the role of information flows tends to be especially underemphasized in global health supply chains (Levine et al. 2008). Although this may be just a consequence of the fact that less information generally is available from developing nations, more research on the value of information in the context of global health supply chains, and on the design of healthy information-generating ecosystems would certainly improve the situation. As of now, in the poorest developing nations, very little information is available on market shares, prices at different stages of the supply chains, and even final, end-user demand. However, before resources are spent correcting this situation, we should have to be able to answer some key questions such as: If we did have more information, what would we do with it? Who should gather what information in a consistent and unbiased manner (Oliva and Watson 2009)? Who should have access to what information? Is more information always better, ceteris paribus? What models best capture the value of information in this context?

\section{Conclusion}

We hope we were able to clearly convey that more work is required to fully understand the dynamics of global health supply chains, and to identify and implement opportunities for improvement. We also wish that some of the preliminary work done by the authors can provide guideposts for future work in this area. We are convinced that our urgent task is to build on the recent progress that health programs world-wide are showing. We are also convinced that operations management reseachers can contribute by applying existing knowledge to global health delivery and by researching new frameworks of analysis that could become the cornerstones for policy advice to those who design, operate, or finance these supply chains. If they could, the millions of people whose health could potentially improve would forever thank this contribution of operations management.

\section{References}

Attridge, C. J., A. S. Preker. 2000. Improving access to medicines in developing countries: Application of new institutional economics to the analysis of manufacturing and distribution issues. HNP Discussion Paper, The World Bank, Washington, DC.

Caldwell, J. C. 1986. Routes to low mortality in developing countries. Popul. Dev. Rev. 12(2): 171-220.

Caldwell, J. C. 1990. Cultural and social factors influencing mortality levels in developing countries. Ann. Am. Acad. Pol. Soc. Sci. 510: 44-59.

Cameron, A., M. Ewen, D. Ross-Degnan, D. Ball, R. Laing. 2009. Medicine prices, availability, and affordability in 36 developing and middle-income countries: A secondary analysis. Lancet 373(9659): 240-249.

Foster, S., R. Laing, B. Melgaard, M. Zaffran. 2006. Ensuring supplies of appropriate drugs and vaccines. Disease Control Priorities in Developing Countries, 2nd edn. Oxford University Press, New York, 1323-1338.

Fundafunda, B., P. Yadav. 2008. Matching demand and supply for pharmaceuticals in a multi-donor environment: The Drug Supply Budget Line (DSBL) in Zambia. Working Paper, William Davidson Institute, University of Michigan, Ann Arbor, MI.

Govindaraj, R., M. Reich, J. Cohen. 2000. World Bank Pharmaceuticals. World Bank, Washington, DC.

Halstead, S. B., J. A. Walsh, K.-N. S. Warren. 1985. Good health at low cost. Rockefeller Foundation, New York.

Kim, K., P. M. Moody. 1992. More resources better health? A cross-national perspective. Soc. Sci. Med. 34(8): 837-842.

Levine, R., J. Pickett, N. Sekhri, P. Yadav. 2008. Demand forecasting for essential medical technologies. Am. J. Law Med. 34: 269-297. 
Macarthur, D. 2007. European pharmaceutical distribution: Key players, challenges and future strategies. Informa UK Ltd., London.

Oliva, R., N. Watson. 2009. Managing functional biases in organizational forecasts: A case study of consensus forecasting in supply chain planning. Prod. Oper. Manag. 18: 2.

Parker, G. G., E. G. Anderson. 2002. From buyer to integrator: The transformation of the supply-chain manager in the vertically disintegrating firm. Prod. Oper. Manag. 11(1): 75-91.

Ravishankar, N., P. Gubbins, R. Cooley, K. Leach-Kemon, C. Michaud, D. Jamison, C. Murray. 2009. Financing of global health: Tracking development assistance for health from 1990 to 2007. Lancet 373(9681): 2113-2124.

Seiter, A. 2010. A Practical Approach to Pharmaceutical Policy. The World Bank, Washington, DC.

Vledder, M., J. Friedman, M. Sjoblom, P. Yadav. 2011. Optimal supply chain structure for distributing essential drugs in low income countries: Results from a quasi-randomized experiment. Working Paper, The World Bank, Washington, DC.

WHO. 2008. 10 Facts on the Global Burden of Disease. Available at http:/ / www.who.int/features/factfiles/global_burden/en/ index.html (accessed date December 29, 2008).

Xu, K., D. B. Evans, G. Carrin, A. M. Aguilar-Rivera, P. Musgrove, T. Evans. 2007. Protecting households from catastrophic health expenditures. Health Aff. 6: 972-983.

Yadav, P. 2010. Improving public health in developing countries through operations research. Cochran, J. J., ed. Wiley Encyclopedia of Operations Research and Management Science, DOI: 10.1002/ 9780470400531.eorms0401.

Yadav, P., H. L. Tata, M. Babaley. 2011. Storage and Supply Chain Management. World Medicines Situation Report 2011. World Health Organization, Geneva. 